\title{
Deterministic and stochastic channel models implemented in a physical layer simulator for Car-to-X communications
}

\author{
J. Nuckelt, M. Schack, and T. Kürner \\ Institut für Nachrichtentechnik, Technische Universität Braunschweig, Germany
}

\begin{abstract}
This paper presents a physical (PHY) layer simulator of the IEEE 802.11p standard for Wireless Access in Vehicular Environments (WAVE). This simulator allows the emulation of data transmission via different radio channels as well as the analysis of the resulting system behavior. The PHY layer simulator is part of an integrated simulation platform including a traffic model to generate realistic mobility of vehicles and a 3D ray-optical model to calculate the multipath propagation channel between transmitter and receiver. Besides deterministic channel modeling by means of ray-optical modeling, the simulator can also be used with stochastic channel models of typical vehicular scenarios. With the aid of this PHY layer simulator and the integrated channel models, the resulting performance of the system in terms of bit and packet error rates of different receiver designs can be analyzed in order to achieve a robust data transmission.
\end{abstract}

\section{Introduction}

Car-to-car or car-to-infrastructure communications - summarized under the term $\mathrm{C} 2 \mathrm{X}$ communications - have been of great research interest in recent years. This technology shall contribute to increase the safety as well as the efficiency of road traffic. The dedicated IEEE standard for vehicular communications is $802.11 \mathrm{p}$, also known as WAVE (IEEE, 2010). The European equivalent is the ETSI standard Intelligent Transportation Systems (ITS)-G5 (ETSI, 2010). Both standards operate in the $5.9 \mathrm{GHz}$ band and are mainly based on the well-known Wireless Local Area Network (WLAN) standard IEEE 802.11a (IEEE, 2007). Since IEEE 802.11a was primarily designed for nomadic indoor usage it has to be

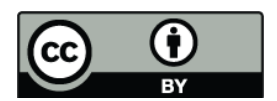

Correspondence to: J. Nuckelt (nuckelt@ifn.ing.tu-bs.de) ensured that $802.11 \mathrm{p}$ will reliably operate in typical vehicular environments with high relative velocities between transmitter and receiver as well as long multipath propagation delays.

In order to investigate and validate the system performance in different $\mathrm{C} 2 \mathrm{X}$ communication scenarios, a PHY layer simulator compliant to IEEE $802.11 \mathrm{p}$ has been developed. In this paper, we give a description of the simulator structure and provide an overview of the implemented channel models. Since the characteristic of the $\mathrm{C} 2 \mathrm{X}$ radio channel has a huge impact on the system performance and each channel model has its advantages and disadvantages, we intend to use different kinds of channel models for the simulations. On the one hand, a self-developed ray-optical tool is used to model the radio channel deterministically. This approach allows us to analyze the system behavior in a specific situation, e.g. two cars are approaching a certain intersection. On the other hand, the stochastic channel models, which can be found in the literature (Acosta-Marum and Ingram, 2007), are used to investigate the system performance in scenarios that represent a certain $\mathrm{C} 2 \mathrm{X}$ communication environment, e.g. a highly mobile highway scenario. In this way, several receiver designs like different channel estimation strategies can be evaluated and compared to each other.

This paper is structured as follows: Sect. 2 gives a detailed description of the developed PHY layer simulator. Section 3 describes the deterministic and stochastic channel models implemented in the simulator. Some performance results in terms of packet error rates are presented in Sect. 4. The paper finally ends with a summary and a conclusion in Sect. 5 .

\section{Description of the PHY layer simulator}

The developed PHY layer simulator is part of an integrated simulation environment for $\mathrm{C} 2 \mathrm{X}$ communications as illustrated in Fig. 1. This simulation platform includes scenario data of the city of Braunschweig, a 3D ray-optical tool to

Published by Copernicus Publications on behalf of the URSI Landesausschuss in der Bundesrepublik Deutschland e.V. 


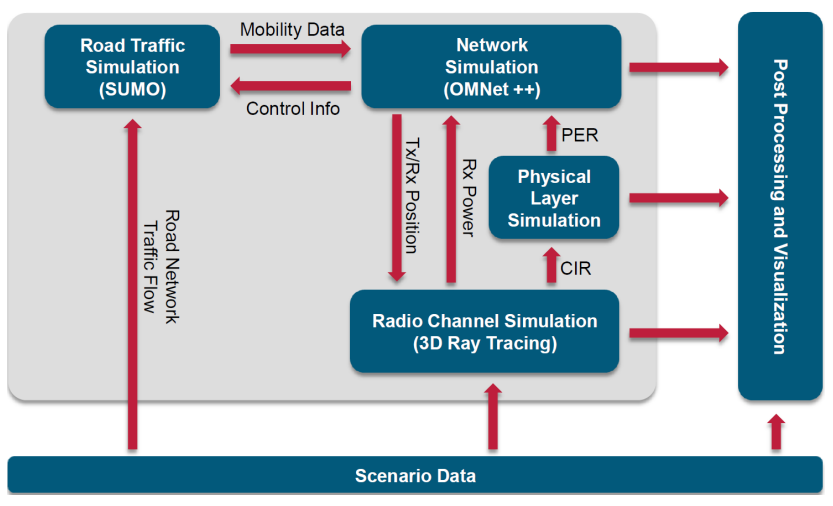

Fig. 1. Integrated simulation environment for $\mathrm{C} 2 \mathrm{X}$ communications.

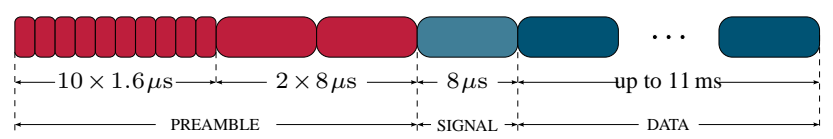

Fig. 2. IEEE 802.11 p frame structure with preamble, signal and data fields.

model the radio channel, a road traffic and network simulator as well as the PHY layer simulator presented in this paper. A more detailed description of the integrated simulation environment can be found in (Schumacher et al., 2009; Kürner and Schack, 2010). Basically, the part of the PHY layer simulator can be divided into three blocks, namely transmitter, radio channel, and receiver. In the following subsections, the structure of these blocks is described in detail.

\subsection{Transmitter}

In IEEE $802.11 \mathrm{p}$, data is transmitted as packets as illustrated in Fig. 2. First, the transmitter creates the Physical Layer Convergence Protocol (PLCP) preamble consisting of 10 short and 2 long training symbols. Depending on the chosen data rate and payload size, the subsequent signal field is generated. After that the payload data is appended. In order to generate the payload, the transmitter creates randomly distributed binary data which thereafter is scrambled using the generator polynomial $S(x)=x^{7}+x^{4}+1$. The output of the scrambler passes the convolutional encoder to introduce a forward error correction (FEC). The generator polynomials of the coder are $g_{0}=133_{8}$ and $g_{1}=171_{8}$ offering a coding rate of $1 / 2$. Coding rates of $2 / 3$ and $3 / 4$ are generated by employing puncturing patterns. The encoded binary data is interleaved by a two-step permutation algorithm. Afterwards, the data stream is modulated to complex symbols, whereas binary phase shift keying (BPSK), quadrature phase shift keying (QPSK), 16- and 64-quadrature amplitude modulation (QAM) are defined by the standard, and is then converted from serial to parallel. The data symbols are mapped to the corresponding subcarriers and four pilot tones are inserted. A 64-IFFT block converts the data from the frequency to the time domain. The IFFT output is parallelto-serial converted and a guard interval in the form of a cyclic prefix $(\mathrm{CP})$ of a period $T_{\mathrm{cp}}=1.6 \mu \mathrm{s}$ is added to overcome the impact of multipath propagation. Several data OFDM symbols as well as the signal field and the preamble are merged to a packet that passes the radio channel block. The complete structure of the transmitter block is illustrated in Fig. 3.

\subsection{Channel}

The time-variant frequency-selective radio channel is modeled by two stages, namely a tapped-delay line model and an additive white Gaussian noise (AWGN) block. First, a discrete convolution of the transmitted packet and channel impulse response (CIR) is performed. The CIR is updated for each sample period, i.e. $T=1 / 10 \mathrm{MHz}=0.1 \mu$ s. The CIR is calculated either by means of 3D ray-optical modeling or derived from the stochastic channel models presented in (Acosta-Marum and Ingram, 2007). The implemented channel models are described in more detail in Sect. 3. Afterwards, AWGN with respect to the selected signal-to-noise ratio (SNR) level is added to the signal. The resulting signal is the input of the receiver block. The block diagram of the radio channel implementation is depicted in Fig. 4. Note that the receiver is also fed with a noiseless version of the signal that has only been convolved with the CIR. In this way, reference information like perfect channel knowledge can be obtained at the receiver.

\subsection{Receiver}

According to the frame structure of IEEE 802.11p, the whole signal processing in the receiver can be divided into two parts: processing of the preamble field and decoding of the signal and data fields. For a better understanding, the structure of the receiver is depicted in Fig. 5. First, the short preamble and the long preamble are used to perform coarse and fine time as well as frequency synchronization. The current version of the implemented simulator performs ideal time synchronization. Coarse and fine frequency synchronization is carried out according to the approach presented in (Terry and Heiskala, 2002). Afterwards, an initial estimation of the channel transfer function (CTF) based on the long preamble is performed.

In the second step, the signal field is equalized using the initial channel estimate and decoded in order to recover the transmitted information about the current data rate and payload size. After that, the OFDM symbols of the data field are decoded. Basically, the receiver performs the inverse signal processing as in the transmitter with some additional steps. First, the CP is removed. The remaining core OFDM symbol is converted from serial to parallel and is transformed to the frequency domain by applying a 64-FFT. After that, a 


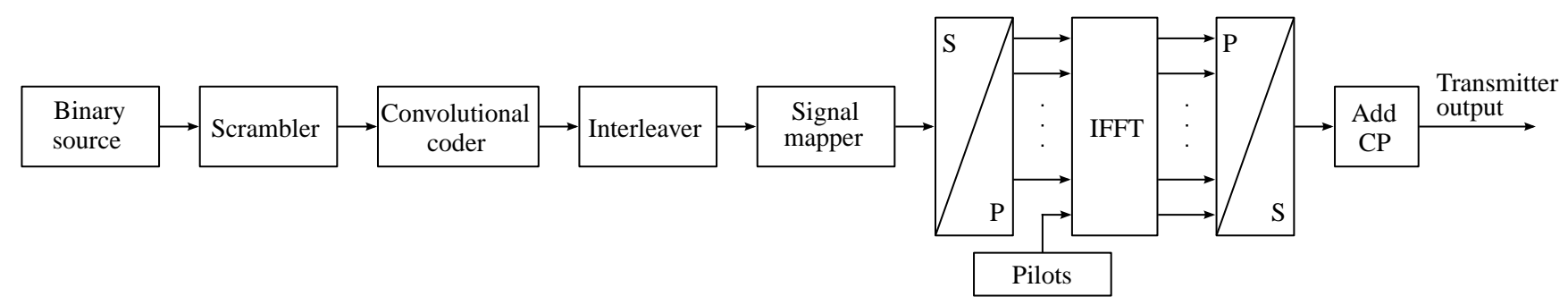

Fig. 3. Block diagram of the transmitter.

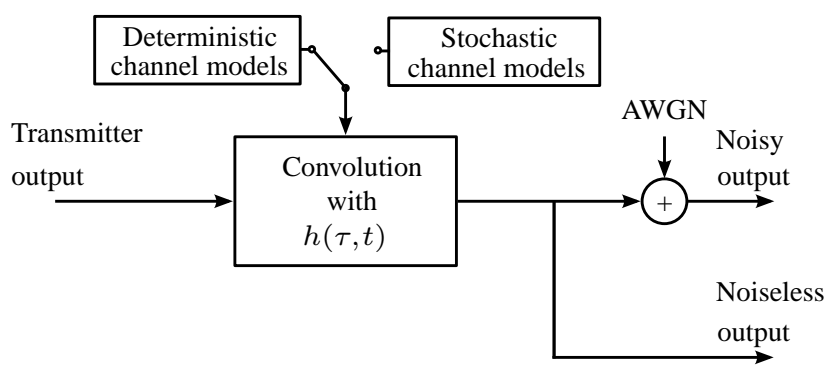

Fig. 4. Block diagram of the radio channel with deterministic and stochastic channel models.

frequency domain channel estimation is performed. On the one hand, the output signal of the channel that is not affected by AWGN can be used to perform an ideal channel estimation. On the other hand, the PHY layer simulator is equipped with an adaptive decision-directed (DD) channel estimation technique as presented in (Skupin et al., 2010). Either the ideally or the realistically estimated CTF is used to perform zero-forcing equalization before the signal is again parallelto-serial converted. In the next step, the complex symbols are demapped to binary data, whereas hard or soft (i.e. approximate log-likelihood ratio (LLR)) decision is performed. Afterwards, the binary data is deinterleaved, decoded by the Viterbi decoder and descrambled. Finally, by a comparison of the originally transmitted data and the decoded received data, the resulting bit and packet error rates are calculated with respect to the current SNR.

In order to validate the results of the PHY layer simulator, the simulated BERs in the AWGN channel have been compared to the theoretical values, whereas both match very well.

\section{Implemented channel models}

Basically, two classes of channel models, namely deterministic and stochastic models, have been implemented in the PHY layer simulator. The concepts of these models are described in this section.

\subsection{Deterministic channel models}

As a deterministic channel model, a 3D ray-optical approach is used in our integrated simulation environment. This model considers the direct path, specular reflections as well as diffuse scattering. Specular reflections are calculated with a ray-tracing model based on the image method (McKown and Hamilton Jr., 1991) up to the $n$-th order, but with respect to the computation time, only first and second order reflections are considered in this investigation. Diffuse scattering is taken into account on surfaces seen by both the transmitter and the receiver. Thus, all surfaces are segmented into tiles and single scattering processes are determined.

For each ray, full-polarimetric antenna patterns are used and channel polarization matrices are computed. The output of the ray-optical model for each communication link is a time-variant CIR $h(\tau, t)$, which completely characterizes the frequency-selective channel and can be expressed in the complex baseband as

$$
\begin{aligned}
h(\tau, t) & =\sum_{k=1}^{N(t)} a_{k}(t) \cdot e^{j\left(2 \pi f \tau_{k}(t)+\varphi_{k}(t)\right)} \cdot \delta\left(\tau-\tau_{k}(t)\right) \\
& =\sum_{k=1}^{N(t)} \underline{a}_{k}(t) \cdot \delta\left(\tau-\tau_{k}(t)\right),
\end{aligned}
$$

where the $k$-th multipath component is described by an amplitude $a_{k}(t)$, a delay $\tau_{k}(t)$ and an additional phase shift $\varphi_{k}(t)$ at time $t$. The number of multipath components, given by $N(t)$, is also time-variant. The amplitude and the phase term can be combined for each multipath component in a complex coefficient $\underline{a}_{k}(t)$, which can be written as

$$
\begin{aligned}
\underline{a}_{k}(t)= & \boldsymbol{e}_{\mathrm{Rx}}\left(\Theta_{\mathrm{Rx}, k}, \Phi_{\mathrm{Rx}, k}\right)^{H} \cdot \mathbf{P}_{k} \\
& \cdot \boldsymbol{e}_{\mathrm{Tx}}\left(\Theta_{\mathrm{Tx}, k}, \Phi_{\mathrm{Tx}, k}\right) \cdot L_{k}(t),
\end{aligned}
$$

where the subscript $H$ denotes the Hermitian transpose. The complex channel polarization matrix $\mathbf{P}_{k}$, which is not normalized and thus includes the reflection or scattering losses of the considered path, is multiplied by the complex polarization vectors $\boldsymbol{e}_{\mathrm{Rx}}\left(\Theta_{\mathrm{Rx}, k}, \Phi_{\mathrm{Rx}, k}\right)$ and $\boldsymbol{e}_{\mathrm{Tx}}\left(\Theta_{\mathrm{Tx}, k}, \Phi_{\mathrm{Tx}, k}\right)$ for the $\mathrm{Rx}$ and $\mathrm{Tx}$ antennas, respectively. These vectors comprise the antenna gains and fully describe the polarization characteristics of the antennas at the angles of arrival $\left(\Theta_{\mathrm{Rx}, k}, \Phi_{\mathrm{Rx}, k}\right)$ 


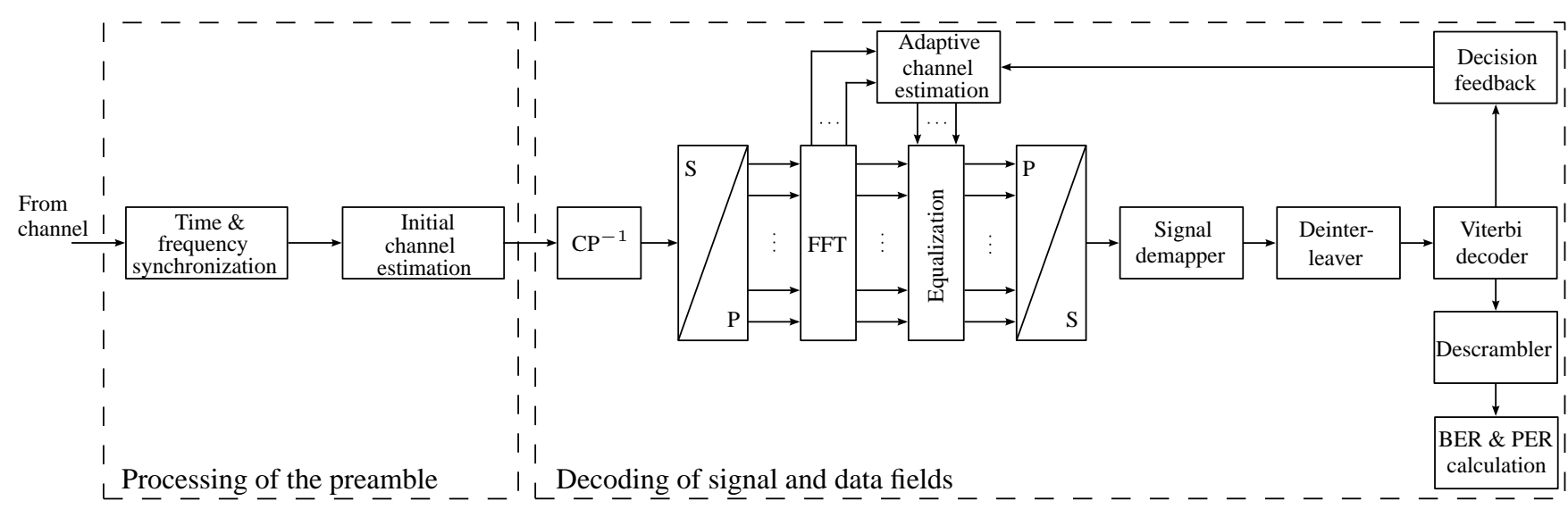

Fig. 5. Block diagram of the receiver.

and the angles of departure $\left(\Theta_{\mathrm{Tx}, k}, \Phi_{\mathrm{Tx}, k}\right)$ for the $k$-th multipath component. The complex factor $L_{k}(t)$ comprises the propagation loss and the phase shift based on the delay $\tau$ of the considered path. For the direct path, $\mathbf{P}_{k}$ is the identity matrix, whereas for reflected and scattered paths of the order $n, \mathbf{P}_{k}$ is calculated according to (Maltsev et al., 2009) using

$$
\begin{aligned}
\mathbf{P}_{k}= & \mathbf{R}\left(\varphi_{\mathrm{Rx}, k}\right) \cdot \prod_{m=0}^{n-2}\left(\mathbf{R}_{n-m, k} \cdot \mathbf{R}\left(\varphi_{n-m, k}\right)\right) \\
& \cdot \mathbf{R}_{1, k} \cdot \mathbf{R}\left(\varphi_{\mathrm{Tx}, k}\right)
\end{aligned}
$$

where

$$
\mathbf{R}(\varphi)=\left[\begin{array}{cc}
\cos \varphi & \sin \varphi \\
-\sin \varphi & \cos \varphi
\end{array}\right]
$$

is the rotation matrix. The value $\varphi_{\mathrm{Tx}, k}$ characterizes the angle between the normal vector of the incidence plane and the field strength component $E_{\theta}$ at the first reflection or scattering point, whereas $\varphi_{\mathrm{Rx}, k}$ is the angle between the scattering or reflecting plane and $E_{\theta}$ at the last reflection or scattering point for the $k$-th multipath component. For multiple reflections or scattering processes, the term $\varphi_{i, k}$ is the angle between $E_{\theta}$ and the normal vector of the incidence plane at the $i$-th reflection or scattering point for the $k$-th multipath component, respectively. The reflection or scattering matrix is given by

$\mathbf{R}_{i, k}=\left[\begin{array}{ll}r_{\perp_{i, k}} & \zeta_{1_{i, k}} \\ \zeta_{2_{i, k}} & r_{\|_{i, k}}\end{array}\right]$,

where the elements $r_{\perp_{i, k}}$ and $r_{\|_{i, k}}$ are the reflection coefficients of the surface belonging to the $i$-th reflection or scattering point of the $k$-th multipath component for perpendicular and parallel components of the electric field, respectively. For reflection processes, these elements are determined based on the Fresnel equations, whereas for scattering processes, the values are calculated using the model of a Lambertian emitter (Degli-Esposti et al., 2007). For the latter, both elements are equal. The elements $\zeta_{1_{i, k}}$ and $\zeta_{2_{i, k}}$ are cross polarization coupling coefficients and are set to zero in this investigation. An extension of this approach for multiple input multiple output (MIMO) systems and an investigation of different antenna configurations can be found in (Schack et al., 2010).

Based on 3D building data, which is available for the city of Braunschweig, and a microscopic road traffic simulator, several traffic scenarios in a dense urban area have been created. These scenarios represent typical traffic situations that are interesting for $\mathrm{C} 2 \mathrm{X}$ applications like intersection collision warning or traffic jam assistants. They can be applied in order to investigate how the underlaying system will operate in specific situations and under different radio channel conditions, e.g. the impact of non-line-of-sight (NLOS) and line-of-sight (LOS). An example scenario with two oncoming cars that cross each other in an urban canyon is depicted in Fig. 6.

The speed of each vehicle is $120 \mathrm{~km} / \mathrm{h}$ and there are LOS conditions at any time.

One great challenge of $\mathrm{C} 2 \mathrm{X}$ communications are the high Doppler shifts due to the relative motion between communicating cars. In this scenario, for example, the relative velocity of transmitter and receiver is $v=240 \mathrm{~km} / \mathrm{h}$ corresponding to a maximum Doppler shift of

$f_{\mathrm{D}, \max }=\frac{v}{c} f_{\mathrm{c}}=1311 \mathrm{~Hz}$

whereas $c$ and $f_{\mathrm{c}}$ denote the speed of light and the carrier frequency of $5.9 \mathrm{GHz}$, respectively. In Fig. 7, the Doppler spectra with respect to the scenario time are depicted. The Doppler shift of the LOS components at $\pm 1311 \mathrm{~Hz}$ can clearly be observed. Besides the dominant LOS component, lower Doppler shifts caused by reflected and scattered waves are also present. The calculated spectra fit very well with the expected ones. 


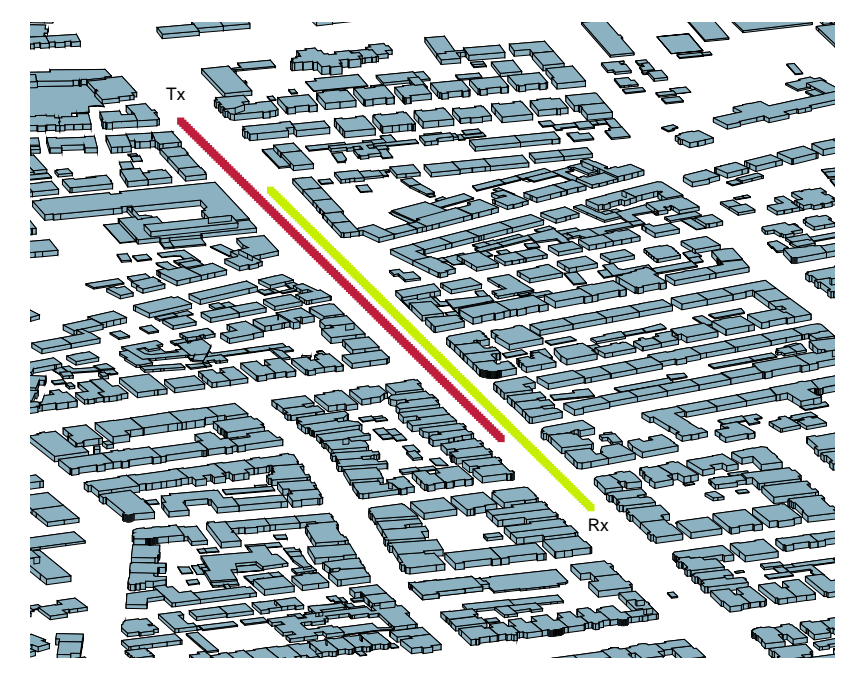

Fig. 6. Exemplary scenario of two oncoming cars in the city of Braunschweig.

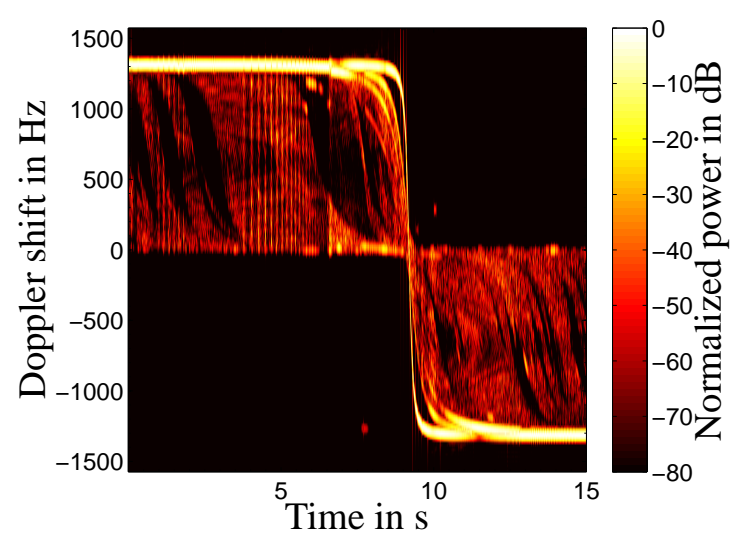

Fig. 7. Calculated Doppler spectra versus scenario time of two oncoming cars.

Furthermore, the corresponding power delay profiles (PDPs) are shown in Fig. 8. As expected, a lot of delayed multipath components due to reflected or scattered waves on surrounding buildings can be observed.

\subsection{Stochastic channel models}

Besides deterministic channel models also six doubly selective stochastic channel models published in (Acosta-Marum and Ingram, 2007) have been implemented. The authors in (Acosta-Marum and Ingram, 2007) present three vehicle-tovehicle (VTV) and three roadside-to-vehicle (RTV) models:

- VTV-Expressway oncoming (EO),

- VTV-Urban canyon oncoming (UCO),

- RTV-Suburban street (SS),

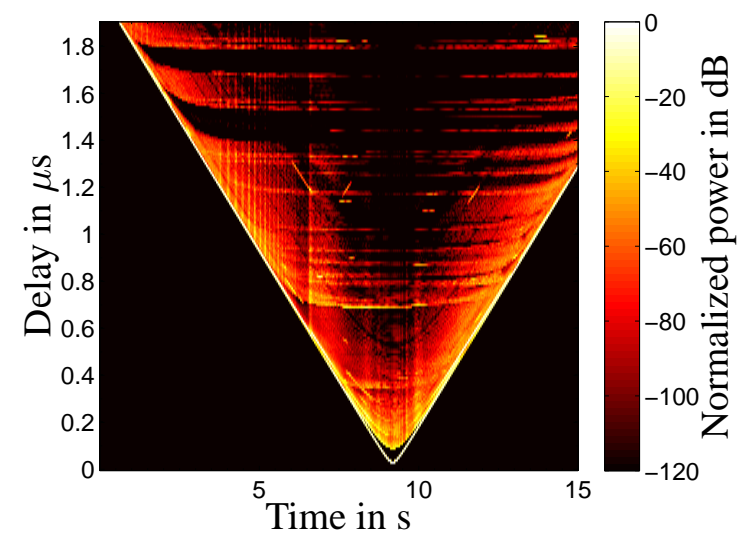

Fig. 8. Power delay profiles of the scenario with oncoming cars.

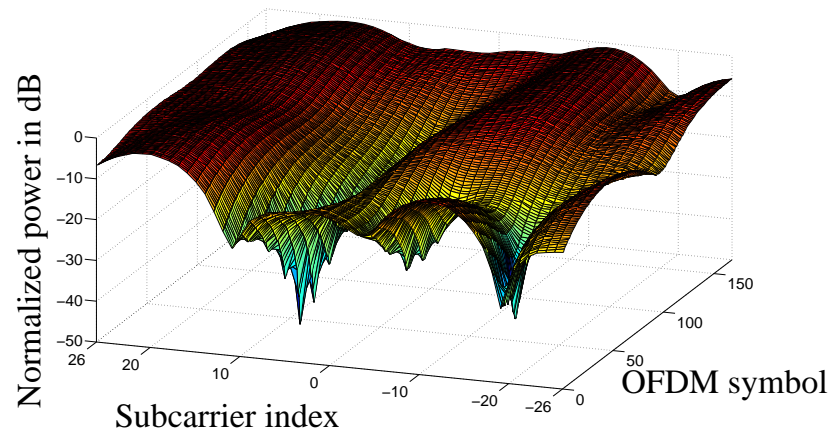

Fig. 9. Realization of a doubly selective channel transfer function of the RTV-SS scenario.

- RTV-Expressway (E) ${ }^{1}$,

- VTV-Expressway same direction with wall (ESDWW) and

- RTV-Urban canyon (UC).

Each scenario is characterized by a more or less dominant LOS component and several superimposed multipath components. All paths are described by a delay value, the relative path loss, a K factor as well as Doppler-related parameters like maximum Doppler shift, LOS Doppler component and the shape of the Doppler spectra. As all Doppler frequencies are scaled to 120 or $140 \mathrm{~km} / \mathrm{h}$, these models can be employed to carry out PHY layer simulations of highly mobile propagation scenarios. A limitation of these channel models is the fact that nonstationarity, which is an important issue for the modeling of $\mathrm{C} 2 \mathrm{X}$ radio channels, is not considered at all. In Fig. 9, a realization of a CTF corresponding to the RTV-SS scenario is exemplarily shown.

The time-variant and frequency-selective character of the typical C2X propagation channel can clearly be observed.

\footnotetext{
${ }^{1}$ According to the note in (Ivan et al., 2010), the $\mathrm{K}$ factor of the first path of this channel model has to be changed to $5.3 \mathrm{~dB}$ (instead of $-5.3 \mathrm{~dB}$ as given).
} 


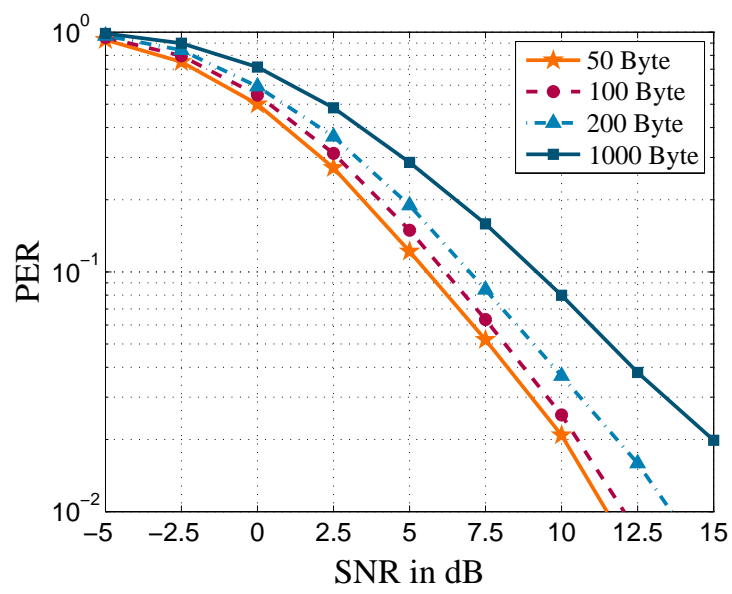

Fig. 10. Impact of the payload size on PER performance in the VTV-EO scenario.

The variation of the radio channel with respect to the time is one of the most challenging issues when designing robust $\mathrm{C} 2 \mathrm{X}$ receivers.

\section{Exemplary simulation results}

This section presents some exemplary simulation results in terms of packet error rates (PERs). The PHY layer of IEEE $802.11 \mathrm{p}$ offers a lot of system parameters that can be modified. For example, the payload size can be chosen up to 4095 byte leading to different packet periods. The length of the transmitted packets has a significant impact on the system performance. In Fig. 10, the PERs of several packet lengths are shown for a data rate of $3 \mathrm{Mbit} / \mathrm{s}$. Perfect channel knowledge was assumed at the receiver and soft-input Viterbi decoding has been performed. The applied channel model is the VTV-EO scenario. It can be observed that the length of a packet has a considerable impact on the resulting performance and, hence, the choice of the payload size has to be carefully decided with respect to the performance requirements of the considered application.

Furthermore, a sophisticated receiver design can also enhance the performance. Different concepts of time and frequency synchronization, channel estimation or diversity techniques have to be investigated on their suitability in $\mathrm{C} 2 \mathrm{X}$ communication systems. For instance, a soft-decision demapper in combination with a preamble-based SNR estimation as it is proposed in (Ren et al., 2009) applied in timevariant and frequency-selective $\mathrm{C} 2 \mathrm{X}$ channel has been compared to simple hard decision. The results in terms of PERs are depicted in Fig. 11. This chart clearly demonstrates that advanced receiver techniques offer a great potential to improve the system performance.

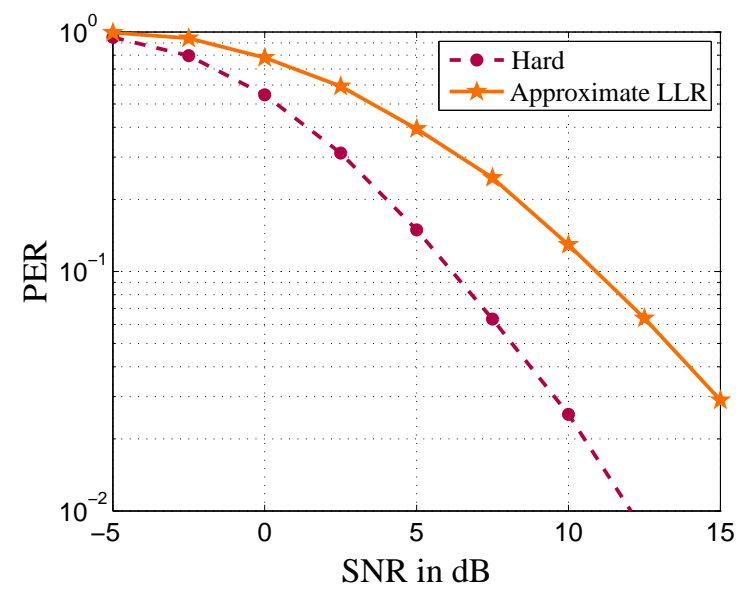

Fig. 11. Performance evaluation of hard and soft (approximate LLR) decision in the VTV-EO scenario.

\section{Conclusions}

This paper presents a PHY layer simulator compliant to the IEEE $802.11 \mathrm{p}$ WAVE standard. This simulator facilitates the analysis of future $\mathrm{C} 2 \mathrm{X}$ communication systems operating in realistic environments. Deterministic channel models by means of 3D ray-optical modeling as well as stochastic channel models derived from an extensive measurement campaign have been implemented in the simulator. In this way, the influence of the fast-fading characteristic of typical $\mathrm{C} 2 \mathrm{X}$ channels on the system performance can be analyzed. Since the radio channel is one of the most challenging issues with respect to PHY layer implementations, different receiver designs can be evaluated in order to develop a robust receiver design with the aid of this simulator.

Future work will focus on PHY layer simulations of the implemented determinstic and stochastic channel models. The results will be evaluated with respect to the advantages and disadvantages of both channel models.

Acknowledgements. The authors are grateful to the Ministry of Science and Culture of Lower Saxony for funding this work within the Connected Cars in a Connected World (C3World) project. Furthermore, they would like to thank Hendrik Hoffmann and Alexander Tschiene for their valuable support.

\section{References}

Acosta-Marum, G. and Ingram, M.: Six time- and frequencyselective empirical channel models for vehicular wireless LANs, Vehicular Technology Magazine, IEEE, 2, 4-11, 2007.

Degli-Esposti, V., Fuschini, F., Vitucci, E. M., and Falciasecca, G.: Measurement and Modelling of Scattering From Buildings, Antennas and Propagation, IEEE Transactions on, 55, 143-153, 2007.

ETSI: Intelligent Transport Systems (ITS); European profile standard for the physical and medium access control layer of Intelli- 
gent Transport Systems operating in the $5 \mathrm{GHz}$ frequency band, ETSI ES 202663 V1.1.0 (2010-01), 2010.

IEEE: IEEE Standard for Information Technology - Telecommunications and information exchange between systems - Local and metropolitan area networks - Specific requirements - Part 11: Wireless LAN Medium Access Control (MAC) and Physical Layer (PHY) Specifications, IEEE Std 802.11-2007 (Revision of IEEE Std 802.11-1999), 2007.

IEEE: IEEE Standard for Information technology - Telecommunications and information exchange between systems - Local and metropolitan area networks - Specific requirements Part 11: Wireless LAN Medium Access Control (MAC) and Physical Layer (PHY) Specifications Amendment 6: Wireless Access in Vehicular Environments, IEEE Std 802.11p-2010, 1-51, 2010.

Ivan, I., Besnier, P., Bunlon, X., Le Danvic, L., Crussiere, M., and Drissi, M.: Influence of propagation channel modeling on V2X physical layer performance, Antennas and Propagation (EuCAP), Proceedings of the Fourth European Conference on, 1-5, 2010.

Kürner, T. and Schack, M.: 3-D Ray-Tracing Embedded Into an Integrated Simulator for Car-to-X Communications, in: URSI Commission B International Symposium on Electromagnetic Theory (EMT-S), Berlin, 2010.
Maltsev, A., Maslennikov, R., Lomayev, A., Sevastyanov, A., and Khoryaev, A.: IEEE 802.11-09/0431r0, 2009.

McKown, J. and Hamilton Jr., R.: Ray tracing as a design tool for radio networks, Network, IEEE, 5, 27-30, 1991.

Ren, G., Zhang, H., and Chang, Y.: SNR estimation algorithm based on the preamble for OFDM systems in frequency selective channels, Communications, IEEE Transactions on, 57, 2230-2234, 2009.

Schack, M., Kornek, D., Slottke, E., and Kürner, T.: Analysis of Channel Parameters for Different Antenna Configurations in Vehicular Environments, Vehicular Technology Conference Fall (VTC 2010-Fall), IEEE 72nd, 1-5, 2010.

Schumacher, H., Schack, M., and Kürner, T.: Coupling of simulators for the investigation of car-to-x communication aspects, in: IEEE Asia-Pacific Services Computing Conference, APSCC, 58-63, 2009.

Skupin, C., Hofmann, F., Garcia, A., and Nuckelt, J.: Adaptive Channel Estimation for VANETs, in: 7th International Workshop on Intelligent Transportation (WIT 2010), 91-96, 2010.

Terry, J. and Heiskala, J.: OFDM Wireless LANs: A Theoretical and Practical Guide, Sams Publishing, Indianapolis, 2002. 